\title{
HBV infection potentiates resistance to S- phase arrest-inducing chemotherapeutics by inhibiting CHK2 pathway in diffuse large B-cell lymphoma
}

Xinying Zhao ${ }^{1,4}$, Xudong Guo ${ }^{2,3}$, Libo Xing ${ }^{2}$, Wenqin Yue ${ }^{1}$, Haisen Yin ${ }^{4}$, Miaoxia He ${ }^{5}$, Jianmin Wang ${ }^{1}$, Jianmin Yang ${ }^{1}$ and Jie Chen ${ }^{1}$

\begin{abstract}
A considerable number of diffuse large B-cell lymphoma (DLBCL) patients are infected with hepatitis B virus (HBV), which is correlated with their poor outcomes. However, the role of HBV infection in $\mathrm{DLBCL}$ treatment failure remains poorly understood. Here, our data demonstrated that HBV infection was closely associated with poorer clinical prognosis independent of its hepatic dysfunction in germinal center B-cell type (GCB type) DLBCL patients. Interestingly, we found that DLBCL cells expressing hepatitis B virus X protein (HBX) did not exhibit enhanced cell growth but did show reduced sensitivity to methotrexate (MTX) and cytarabine (Ara-C), which induced S-phase arrest. Mechanism studies showed that HBX specifically inhibited the phosphorylation of checkpoint kinase 2 (CHK2, a key DNA damage response protein). CHK2 depletion similarly conferred resistance to the S-phase arrest-inducing chemotherapeutics, consistent with HBX overexpression in DLBCL cells. Moreover, overexpression of wild-type CHK2 rather than its unphosphorylated mutant (T68A) significantly restored the reduced chemosensitivity in HBX-expressing cells, suggesting that HBV infection conferred resistance to chemotherapeutics that induced S-phase arrest by specifically inhibiting the activation of CHK2 response signaling in DLBCL.
\end{abstract}

\section{Introduction}

Diffuse large B-cell lymphoma (DLBCL), which accounts for $30-40 \%$ of non-Hodgkin lymphoma (NHL), is an aggressive disease featuring heterogeneous genetic, phenotypic, and clinical characteristics ${ }^{1}$. The combination of rituximab and CHOP (cyclophosphamide, doxorubicin, vincristine, and prednisone) has dramatically improved the outcome in DLBCL patients, with the 3-year event-

\footnotetext{
Correspondence: Jie Chen (chenjiedoctor@163.com)

'Department of Hematology, Changhai Hospital, Second Military Medical University, Shanghai, China

${ }^{2}$ Clinical and Translational Research Center of Shanghai First Maternity and Infant Health Hospital, Shanghai Key Laboratory of Signaling and Disease Research, Collaborative Innovation Center for Brain Science, School of Life Science and Technology, Tongji University, Shanghai, China Full list of author information is available at the end of the article Xinying Zhao and Xudong Guo contributed equally to this work. Edited by $\mathrm{H}-\mathrm{U}$ Simon.
}

free survival ranging from 65 to $80 \%^{2}$. However, approximately $30-40 \%$ of DLBCL patients will develop relapsed or refractory, which remains the major cause of mortality ${ }^{3}$. Thus, it is imperative to investigate the mechanisms of relapse and refractory to develop better chemotherapy options.

Virus infection is considered as a critical inducer of various diseases, especially cancer. Epstein-Barr virus has been proven to associate with the occurrence and progression of nasopharyngeal carcinoma and $\mathrm{NHL}^{4}$. Hepatitis $\mathrm{C}$ virus and hepatitis $\mathrm{B}$ virus (HBV) are also able to promote $\mathrm{NHL}^{5}$. HBV has infected 350 million people worldwide and is responsible for 340,000 cases of liver cancer and 500,000 to 1.2 million liver-related deaths annually. In addition, epidemiologic investigations in 
HBV endemic areas, including China, South Korea, and Japan, show that HBV infection is more common in NHL patients compared with the general population (19.94\% vs. $7.18 \%)^{6-8}$. Importantly, hepatitis B surface antigen (HBsAg)-positive DLBCL patients display poorer chemotherapy responses and shorter progression-free survival (PFS) and overall survival (OS) compared with HBsAg-negative patients as an independent prognostic factor in DLBCL ${ }^{6,9}$. These reports mainly focus on investigating the clinical correlation between HBV infection and poor prognosis of DLBCL, but the exact mechanisms remain unexplored. Hepatitis $\mathrm{B}$ virus $\mathrm{X}$ protein (HBX), has been reported to induce hepatocarcinogenesis by interacting with various signal transduction pathways, such as the $\operatorname{SIRT}^{10}, \mathrm{Wnt} / \beta$-catenin ${ }^{11}$, signal transducer and activator of transcription ${ }^{12}$, and nuclear factor- $\mathrm{KB}$ pathway ${ }^{13}$ to induce hepatocarcinogenesis. The lymphotropic characteristic of HBV renders the HBX detectable in lymphoma tissue ${ }^{14-17}$. However, the role of $\mathrm{HBX}$ in causing progression and poor outcome in DLBCL patients has not been extensively studied.

Chemoresistance is the major cause of treatment failure, which results in relapsed and refractory DLBCL. The activation of the DNA damage response (DDR) allows cells self-repair to resist external damage via activating the downstream cyclins and apoptotic proteins, which results in chemoresistance ${ }^{18}$. DDR suppression is believed to sensitize tumor cells to chemotherapeutic treatments by causing cell death or senescence in the absence of checkpoints and efficient DNA repair ${ }^{19-23}$. On the other hand, DDR inhibition attenuates the cell cycle arrest that occurs during self-repair, which can reduce sensitivity to chemotherapeutic $^{24,25}$. The role of DDR signals in the chemoresistance of DLBCL patients with HBV infection is still unclear.

After retrospectively analyzing 428 DLBCL patients, we found that HBV infection was closely associated with reduced responses to chemotherapy and poor OS and PFS of DLBCL patients, especially in germinal center B-cell type (GCB type) independent of its liver damage. Our results also showed that HBX conferred resistance to chemotherapeutics that induced S-phase arrest by specifically blocking the activation of checkpoint kinase 2 (CHK2) signaling in vitro and in vivo, which explained the poor outcome caused by HBV infection in DLBCL patients.

\section{Results}

HBV infection is correlated with poor prognosis of GCBtype DLBCL patients independent of its liver-damaging effects

We examined 428 DLBCL patients from January 2004 to July 2014 in Changhai Hospital (Shanghai, China) for HBsAg, and 93 cases were HBsAg positive. The HBV infection rate was much higher in DLBCL patients (21.7\%) than that in the general population $(7.18 \%)^{6}$. The clinical and pathological characteristics of DLBCL patients with or without HBV infection were shown in Table 1 . The proportion of male patients with HBV infection (69.9\%) was significantly higher than that without HBV infection (56.1\%) $(P=0.017$, Table 1$)$. HBV-positive patients tended to be younger than HBV-negative patients (median age, 47.0 vs. $55.0, P=0.032$ ). There were no differences in cell of origin, Ki67 expression, lactate dehydrogenase (LDH), and proportions of rituximab included chemotherapy regimens between two groups, but HBV infection was closely associated with more advanced stages $(P=0.014$, Table 1$)$ and increased frequency of treatment failure $(P=0.028$, Table 1$)$, indirectly suggesting that HBV infection contributed to poor outcome.

Kaplan-Meier analysis of the HBV-positive and HBVnegative cohorts revealed that HBV infection had a profound negative impact on the prognosis of DLBCL patients. Patients with HBV infection had significantly shorter OS and PFS than HBV-negative patients (Fig. 1a). After stratifying all the patients into GCB and non-germinal center Bcell (non-GCB) types, we found that DLBCL patients with HBV infection had significantly shorter OS in GCB type, but not non-GCB type, suggesting HBV infection mainly influenced the poor prognosis of GCB-type DLBCL patients (Figs. 1b, c). Increased hepatitis indicators, including alanine aminotransferase, aspartate aminotransferase, gammaglutamyl transpeptidase, albumin, total bilirubin, and direct bilirubin, were more frequently observed in whole course of HBsAg-positive subjects (Supplementary Fig. 1a), in spite that they received anti-viral treatment before chemotherapy until 6 months after chemotherapy. We surprisingly found that abnormal liver function showed no significant impact on OS and PFS of the whole group, the HBV-positive cohort, or the HBV-negative cohort (Figs. 1d-f). In accordance with previous studies ${ }^{14-17}$, the HBX protein could be detected in lymphoid tissues of HBV-infected patients (Supplementary Fig. 1b). Our results thus indicated that HBV infection might reduce the OS and PFS of DLBCL patients by directly affecting the biological properties of DLBCL cells independent of its liver-damaging effects.

\section{HBX attenuates the proliferation inhibition of chemotherapeutics that induce S-phase arrest}

According to our clinical data analysis, the DLBCL patients with GCB-type HBV infection exerted shorter OS than that without HBV infection (Fig. 1c). We then focus on investigating the exact effects of HBX on the biological properties of GCB-type DLBCL cells. To analyze the effect of HBX on DLBCL cell growth, HBX was ectopically overexpressed in GCB-type DLBCL cell lines (SUDHL-4 and $\mathrm{DB}$ ), which was verified by quantitative polymerase chain reaction (qPCR) and western blotting (Figs. 2a, b). However, HBX-expressing DLBCL cells did not exhibit 
Table 1 Characteristics of HBsAg-positive and HBsAg-negative subjects in Changhai Hospital ( $n=428)$

\begin{tabular}{|c|c|c|c|}
\hline Characteristics, $n$ (\%) & $\operatorname{HBV}(-), n=335(78.3 \%)$ & $\operatorname{HBV}(+), n=93(21.7 \%)$ & $P$-value \\
\hline Gender & & & 0.017 \\
\hline Male & $188(56.1 \%)$ & $65(69.9 \%)$ & \\
\hline Female & 147 (43.9\%) & $28(30.1 \%)$ & \\
\hline Median age & 55.0 & 47.0 & 0.032 \\
\hline Stage & & & 0.014 \\
\hline $\mid+\|$ & $130(38.8 \%)$ & $23(24.7 \%)$ & \\
\hline III+IV & $205(61.2 \%)$ & $70(75.3 \%)$ & \\
\hline Cell of origin & & & 0.598 \\
\hline Non-GCB & $190(56.7 \%)$ & $57(61.3 \%)$ & \\
\hline GCB & $97(29.0 \%)$ & $25(26.9 \%)$ & \\
\hline Not available & $48(14.3 \%)$ & $11(11.8 \%)$ & \\
\hline Liver function & & & 0.002 \\
\hline Normal & $320(95.5 \%)$ & $79(84.9 \%)$ & \\
\hline Abnormal & $15(4.5 \%)$ & $14(15.1 \%)$ & \\
\hline $\mathrm{LDH}$ & & & 0.082 \\
\hline Normal & $231(69.0 \%)$ & $55(59.1 \%)$ & \\
\hline Elevated & $104(31.0 \%)$ & $38(40.9 \%)$ & \\
\hline Median Ki67 expression & $71.85 \%$ & $70.00 \%$ & 0.833 \\
\hline Chemotherapy outcome & & & 0.028 \\
\hline $\mathrm{CR}+\mathrm{PR}$ & $246(73.4 \%)$ & $57(61.3 \%)$ & \\
\hline Relapse+NR & $89(26.6 \%)$ & $36(38.7 \%)$ & \\
\hline Rituximab included & & & 0.069 \\
\hline No & 118 (35.2\%) & $43(46.2 \%)$ & \\
\hline Yes & $217(64.8 \%)$ & $50(53.8 \%)$ & \\
\hline
\end{tabular}

enhanced proliferation or differences in cell cycle distribution compared with control cells (Supplementary Figs. $2 \mathrm{a}$ and $\mathrm{b}$ ). There was also no significant difference in the expression of cell cycle-related proteins upon exogenous expression of HBX compared with control cells (Supplementary Figs. 2c and d). Neither control cells nor HBX-expressing cells exhibited obvious apoptosis, as evaluated by flow cytometry (Supplementary Fig. 2e). These data suggested that HBX had no significant effect on the proliferation, cell cycle distribution, or apoptosis of DLBCL cells.

We further sought to investigate the effect of HBX on the response to chemotherapeutics. We tested the growth of control and HBX-expressing cells treated with the chemotherapeutic agents methotrexate (MTX), cytarabine (Ara-C), vindesine (VDS), and epirubicin (EPI) at a concentration of the IC50 value for 24,48 , and $72 \mathrm{~h}$. Compared with control cells, SUDHL-4 and DB cells overexpressing HBX showed enhanced resistance to MTX and Ara-C after treatment for 48 and $72 \mathrm{~h}$ (Figs. 2c, d), whereas there was no significant difference in proliferation inhibition upon treatment with VDS and EPI (Fig. 2e). Furthermore, the cell cycle distributions analysis of control and HBX-expressing cells treated with or without MTX or Ara-C, which were characterized by inducing S-phase arrest, showed that HBX overexpression significantly attenuated the S-phase arrest in both SUDHL-4 and DB cells upon treatment with MTX and Ara-C (Figs. 2f, g). These findings indicated that HBX prevented the cytotoxicity and impaired the S-phase arrest of chemotherapeutics, which might explain why HBV infection cause poor outcome in DLBCL patients.

\section{HBX specifically inhibits CHK2 phosphorylation}

DDR has been reported to be intimately correlated with chemoresistance $^{26}$. Phosphorylation of H2AX at Ser139 $(\gamma \mathrm{H} 2 \mathrm{AX})$ is one of the early response to DNA damage, which is considered as a sensitive biomarker for $\mathrm{DDR}^{27,28}$. 


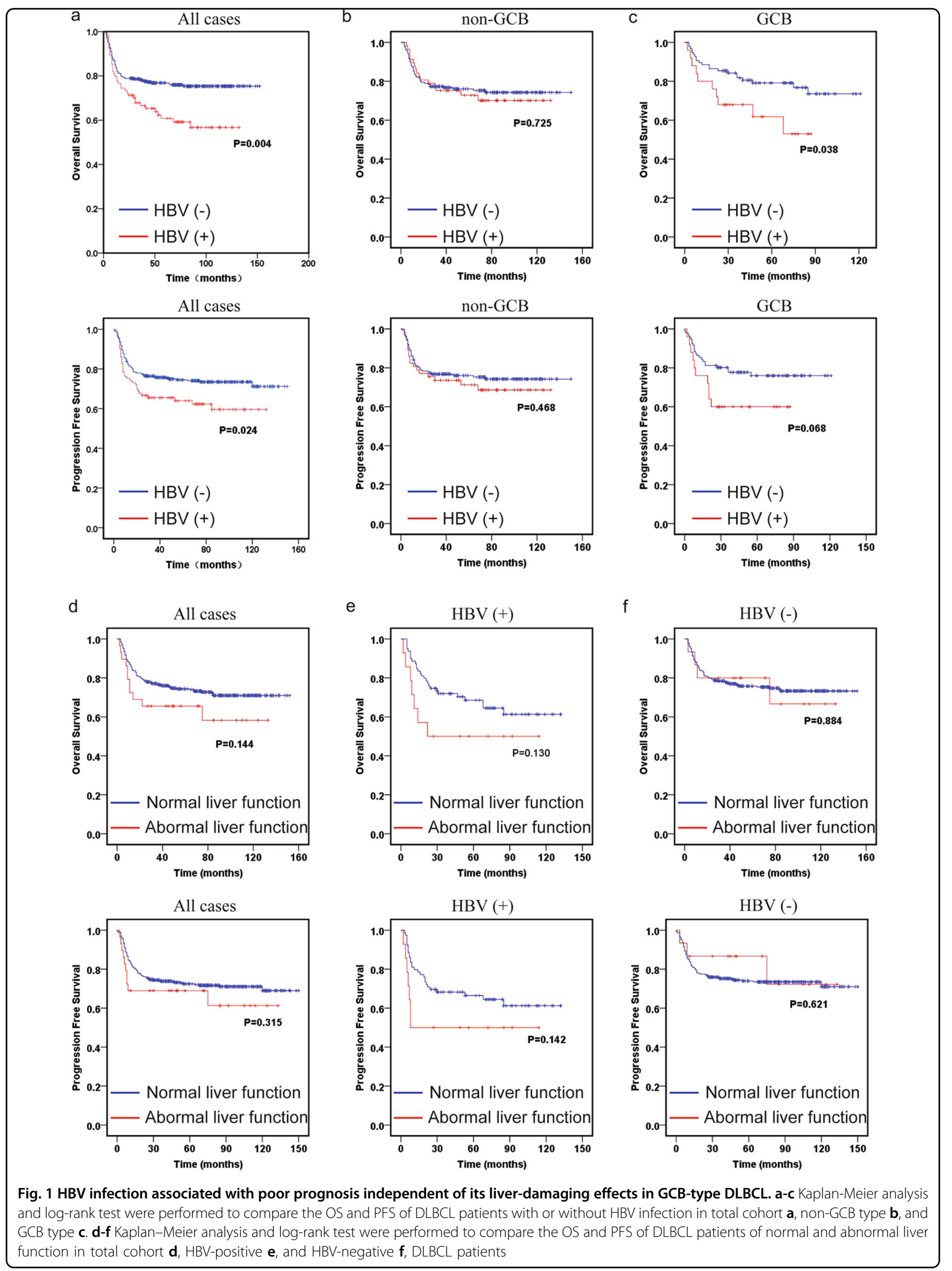




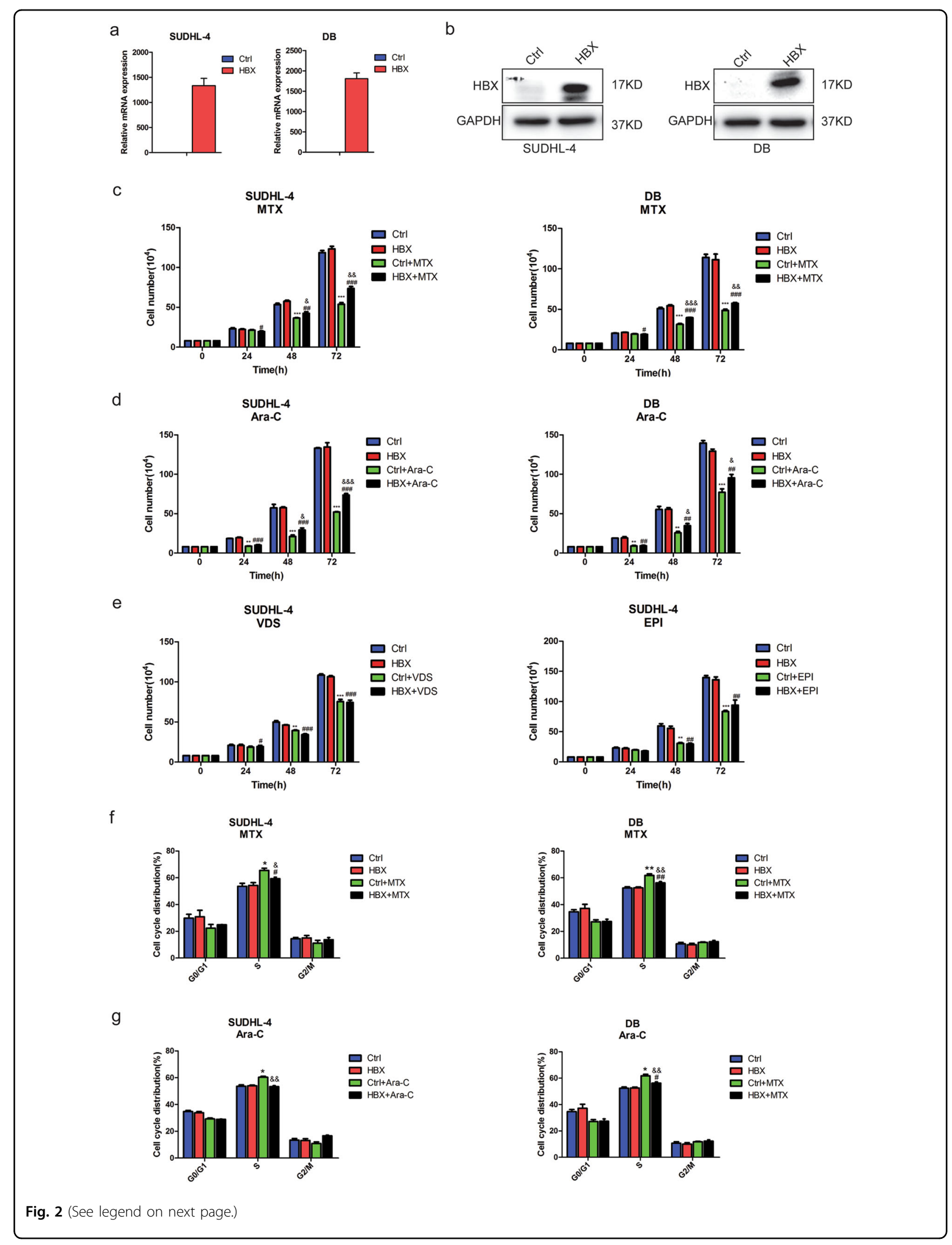


(see figure on previous page)

Fig. $2 \mathrm{HBX}$ attenuates the proliferation inhibition of chemotherapeutics that induce S-phase arrest. $\mathbf{a}, \mathbf{b}$ Ectopic overexpression of HBX in SUDHL-4 and DB cells was determined by $\mathrm{qPCR} \mathbf{a}$, and western blotting b. c, d Cell-counting assay for control or HBX-expressing SUDHL-4 and DB cells treated with MTX $(4 \mathrm{ng} / \mathrm{ml})$ or Ara-C $(20 \mathrm{ng} / \mathrm{ml})$; the cell numbers were measured at 24,48 , and $72 \mathrm{~h}$. e Cell-counting assay for control or HBXexpressing SUDHL cells treated with VDS $(0.6 \mathrm{ng} / \mathrm{ml})$ or EPI $(100 \mathrm{ng} / \mathrm{ml})$; the cell numbers were measured at 24,48 , and $72 \mathrm{~h}$. $\mathbf{f}, \mathbf{g}$ Quantification of the cell cycle distributions in control and HBX-expressing SUDHL-4 and DB cells treated with MTX $\mathbf{f}$, or Ara-C $\mathbf{g}$, for $48 \mathrm{~h}$. These results are shown as the mean \pm SEM from triplicate experiments. " "*" Represents Ctrl + agents versus Ctrl: ${ }^{*} P<0.05$, ${ }^{* *} P<0.01,{ }^{* *} P<0.001$. "\#" Represents HBX + agents versus

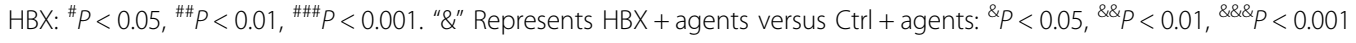

We found that HBX-expressing cells presented lower expression of $\gamma \mathrm{H} 2 \mathrm{AX}$ than control cells after treated with MTX, indicating that HBX expression attenuated the chemotherapeutic-induced DNA damage in DLBCL cells (Figs. 3a, b). To gain mechanistic insight into the molecules potentially involved in the decreased chemosensitivity resulting from $\mathrm{HBV}$ infection, alterations in two major DDR pathways ataxia-telangiectasia mutated (ATM)/CHK2, and ATM and Rad-3 related (ATR)/ checkpoint kinase 1 (CHK1) were assessed. The expression level of P-CHK1 was increased after treatment with MTX for $48 \mathrm{~h}$ in both control and HBX-expressing cells, but neither the baseline nor the post-treatment level of $\mathrm{P}$ CHK1 was affected by overexpressing HBX. Unexpectedly, the expression level of P-CHK2 in control cells was markedly increased to a greater extent than that in HBXexpressing cells after treatment with MTX for $48 \mathrm{~h}$, whereas no significant difference was observed in the baseline P-CHK2 level between control and HBXexpressing cells (Figs. 3c, d). Both the mRNA and protein levels of CHK1, CHK2, and ATM (an upstream kinase of CHK2) were largely unchanged, indicating that HBX might specifically inhibit the phosphorylation of CHK2 (Figs. 3e, f). Activated CHK2 signaling induced cell cycle arrest through activating P53 followed by P21 ${ }^{29}$. Our results showed that the expression levels of P53 and P21, consistent with the expression level of P-CHK2, were less increased in HBX-overexpressing cells after treatment with MTX than that in control cells (Figs. 3g, h). Together, these results indicated that HBX overexpression appeared to specifically inhibit CHK2 phosphorylation caused by MTX treatment in DLBCL cells.

\section{CHK2 phosphorylation rescues chemosensitivity in HBX- expressing cells}

To investigate the functional role of $\mathrm{P}-\mathrm{CHK} 2$ in chemoresistance, we attempted to downregulate CHK2 in SUDHL-4 cells using short hairpin RNA (shRNA). CHK2 depletion led to reduced expression of both P-CHK2 and CHK2 (Fig. 4a). The increase in P-CHK2 was less pronounced in shCHK2-1 and shCHK2-2 cells with MTX treatment compared with control cells (Fig. 4a). We further found that CHK2-depleted cells exhibited normal cell growth compared to control cells, consistent with previous reports (Fig. 4b) ${ }^{30}$. CHK2-depleted cells displayed a modest proliferation decrease and S-phase increase after MTX treatment for $48 \mathrm{~h}$, which simulated the effects of HBX overexpression on proliferation and the proportion of cells in S-phase (Figs. 4c, d). As downregulation of CHK2 could result in enhanced chemoresistance after MTX treatment, we tried to reverse the reduced chemosensitivity of HBX-expressing cells by overexpressing wild-type CHK2 (WT) or an unphosphorylated CHK2 mutant (T68A) (Fig. 4e). An increase in the CHK2 level was detected after overexpressing both CHK2 (WT) and CHK2 (T68A), whereas increased expression of P-CHK2 was only observed after CHK2 (WT) overexpression (Fig. 4f). As expected, overexpressing CHK2 (WT) rather than mutant CHK2 (T68A) reversed the reduced proliferation inhibition and decreased S-phase arrest caused by HBX overexpression after MTX treatment (Figs. 4g, h). These results indicated that CHK2 could overcome HBXinduced chemoresistance and that this effect was dependent on its phosphorylation.

\section{HBX confers resistance to chemotherapy in vivo}

To further assess HBV infection as a mediator of chemoresistance, we examined the inhibition effect of MTX on control and HBX-expressing cells in vivo. Control and HBX-overexpressing DB cells were grafted into the nonobese diabetic-severe combined immunodeficiency disease (NOD-SCID) mice. Two weeks after injection, the mice were treated with MTX $(10 \mathrm{mg} / \mathrm{kg}$ by intraperitoneal injection every 3 days), and tumor sizes were measured (Fig. 5a). MTX treatment significantly reduced the tumor burden of mice injected with control cells compared with untreated mice (Figs. 5b, c). However, the tumor burden in mice injected with DB cells expressing HBX was not reduced as effectively as that in mice injected with control cells (Figs. 5b, c). The tumor specimens were then subjected to immunohistochemical staining to analyze the expression of DDR proteins, and our data showed that the expression level of $\mathrm{P}-\mathrm{CHK} 2$ was mildly increased in tumors derived from HBX-expressing cells compared with those derived from control cells, whereas the increase extent of P-CHK1 was not affected by HBX overexpression (Figs. 5d, e). Consistent with the results in vitro, the expression levels of CHK2, ATM, and CHK1 were largely unchanged in both control and HBXexpressing cells with or without MTX treatment 


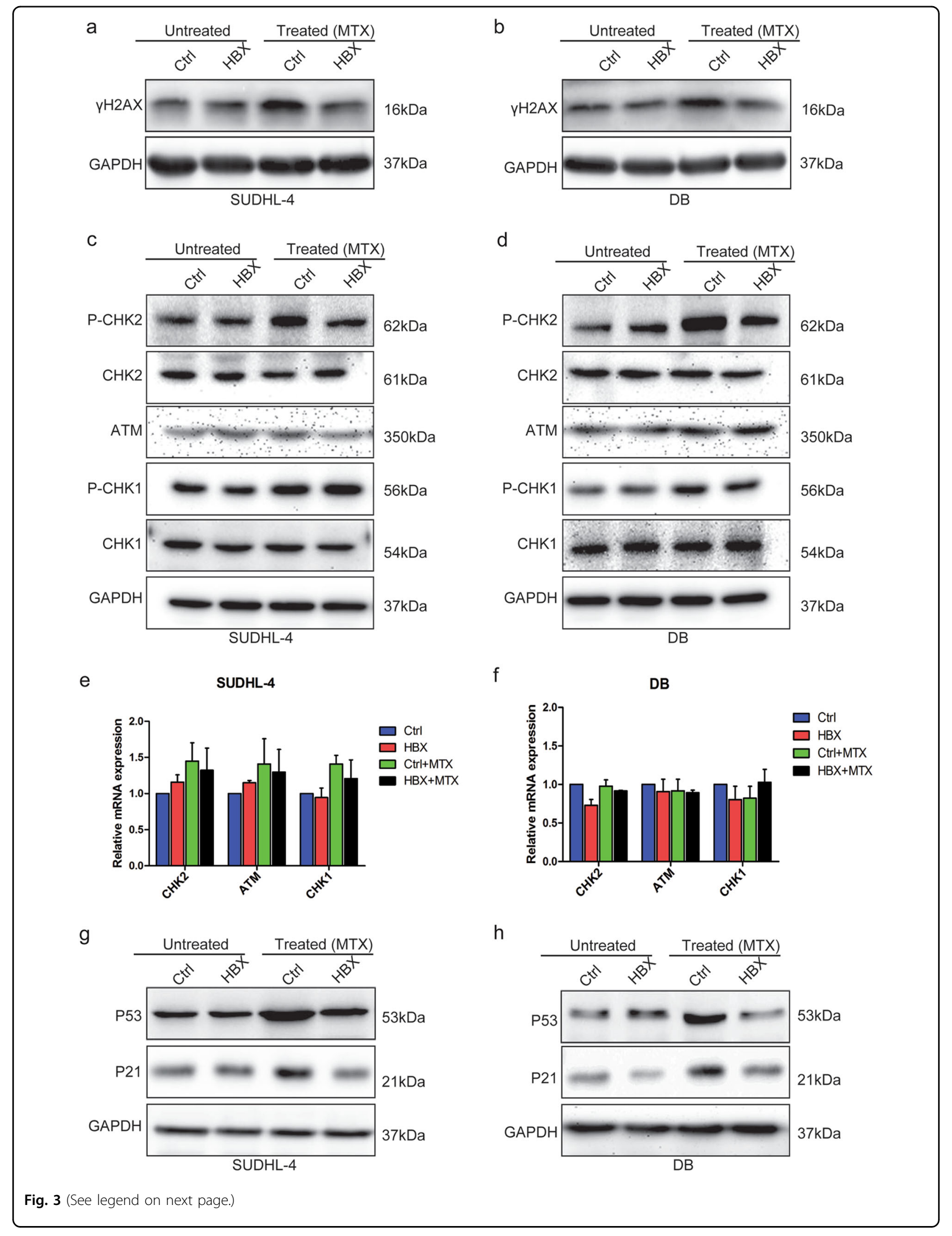


(see figure on previous page)

Fig. 3 HBX specifically inhibits CHK2 phosphorylation. $\mathbf{a}$, $\mathbf{b}$ Western blotting for $\mathrm{\gamma H} 2 \mathrm{AX}$ in control and HBX-expressing SUDHL-4 a, and DB $\mathbf{b}$, cells with or without MTX treatment ( $4 \mathrm{ng} / \mathrm{ml}$ ) for $48 \mathrm{~h}$. $\mathbf{c}$, $\mathbf{d}$ Western blotting for DDR proteins (P-CHK2/CHK2/ATM and P-CHK1/CHK1) in control and HBXexpressing SUDHL-4 $\mathbf{c}$, and DB $\mathbf{d}$, cells with or without MTX treatment $(4 \mathrm{ng} / \mathrm{ml})$ for $48 \mathrm{~h}$. e, $\mathbf{f}$ The mRNA levels of DDR proteins (ATM, CHK2, and CHK1) were largely unchanged in control and HBX-expressing SUDHL-4 e, and DB f $\mathbf{f}$, cells with or without MTX treatment. $\mathbf{g}, \mathbf{h}$ The expression of the P-CHK2 downstream genes P53 and P21 in control and HBX-expressing SUDHL-4 ga and DB $\mathbf{h}$, cells with or without MTX treatment. The results are shown as the mean \pm SEM from triplicate experiments. GAPDH was used as a loading control

(Figs. 5d, e). These in vivo findings showed that HBV infection was a potent mediator of resistance to chemotherapeutics that induce S-phase arrest.

\section{Discussion}

Large-scale epidemiological investigations have suggested that $\mathrm{HBV}$ infection is positively correlated with the onset and progression of NHL. A large cohort study enrolling 603,585 individuals (53,045 HBV positive) in Korea indicated that the HBV-positive population had an increased risk of developing DLBCL throughout 14 years of follow-up ${ }^{31}$. It has also been reported that HBsAg-positive DLBCL patients exhibit more advanced disease stages, lower response to chemotherapy, and worse outcomes compared with HBsAg-negative DLBCL patients ${ }^{5,9}$. HBV-infected DLBCL patients are more likely to experience liver damage, while it is still conflicted that whether hepatic dysfunction serves as a prognosis factor for DLBCL during chemotherapy ${ }^{6,9}$. Our data demonstrated that HBsAg-positive DLBCL patients especially GCB type had lower chemotherapy efficacy and poorer outcomes than HBsAg-negative patients and that HBV-induced chemoresistance, but not hepatic dysfunction, contributed to the poor outcome.

Chemoresistance is widely known as a major cause of treatment failure ${ }^{18,32-34}$. HBV-infected DLBCL patients are more likely to exhibit advanced disease stages, which often preclude the first-line chemotherapies, including $\mathrm{CHOP}$ and R-CHOP. Our results showed that overexpression of the HBX protein resulted in resistance to the S-phase arrest-inducing chemotherapeutics (MTX and Ara-C) rather than two other drugs (VDS and EPI) in DLBCL cells, which was further confirmed by the impaired S-phase arrest in HBX-overexpressing DLBCLs treated with MTX or Ara-C. Thus, our findings indicated that HBV infection might result in lower sensitivity to MTX- or Ara-C-based second-line chemotherapy regimens including ESHARP (etoposide, Ara-C and prednisone), MA (MTX and Ara-C), DHAP (cisplatin, Ara-C and prednisone,and CODOX-MTX (cyclophosphamide, VDS, doxorubicin, and HD-MTX). Further investigations of autologous hematopoietic hepatocyte transplantation ${ }^{35}$ and other intensive chemotherapies, including R-EPOCH (rituximab, etoposide, cyclophosphamide, doxorubicin, vincristine, and prednisone $)^{36,37}$, which have shown favorable effects in the treatment of aggressive DLBCL, might be of great benefit to DLBCL patients infected with
HBV. Several studies have indicated that HBV infection results in the onset and progression of hepatocellular carcinoma because of its hepatotropic peculiarity ${ }^{38,39}$. HBV-DNA is consisted of four segments ( $, P, C$, and $\mathrm{X}$ ). HBX, coded by HBV-X segment, is considered as a small "transcriptional activator" that stimulates transcription by activating Ras-Raf-mitogen-activated protein kinase (MAPK) signaling cascade and promotes cell proliferation by deregulating cell cycle checkpoint controls, then contributes to viral carcinogenesis ${ }^{40,41}$. HBX was also reported to promote hepatocarcinogenesis by regulating various signals ${ }^{10-12 .}$ However, our present data found that HBX had no effects on the cell growth, cell cycle distribution, or apoptosis of DLBCL cells, but reduced the chemosensitivity to drugs that induce S-phase arrest. The roles of other HBV segments in the DLBCL cell property needed to be further explored. Collectively, our study indicated a new function of HBX in regulating DLBCL cell chemoresistance and suggested that HBsAg-positive DLBCL patients would benefit less from MTX- or AraC-based chemotherapy than HBsAg-negative ones.

Much work has demonstrated that DDR is closely related to chemoresistance ${ }^{18,42,43}$. ATM/CHK2 and ATR/ CHK1, two key pathways in DDR signaling, are activated upon DNA damage, leading to cell cycle arrest ${ }^{19-23}$. Our results showed that both MTX- and Ara-C-induced DNA damage dramatically activated CHK2 signaling and caused S-phase arrest to inhibit DLBCL cell growth. HBX overexpression significantly attenuated the activation of CHK2 signaling induced by S-phase arrest drugs, together with its downstream proteins P53 and P21, but did not affect the CHK1 pathway. CHK2 knockdown mimicked HBX-induced resistance to the S-phase arrest-inducing agents, and CHK2 overexpression overcame the resistance caused by HBX overexpression, indicating that CHK2 might serve as a potential mediator of HBXinduced chemoresistance and its de-phosphorylation caused by HBX impaired the S-phase arrest via inhibiting the downstream P53-P21 pathway in DLBCL. Furthermore, our present data showed that the chemosensitivity of HBX-expressing cells could be rescued by overexpressing wild-type CHK2, but not the unphosphorylated CHK2 mutant (T68A). HBX has previously been reported to modulate the phosphorylation of phosphatidylinositol 3 kinase/AKT and MAPK/extracellular signal-regulated kinase signals in liver and 
a

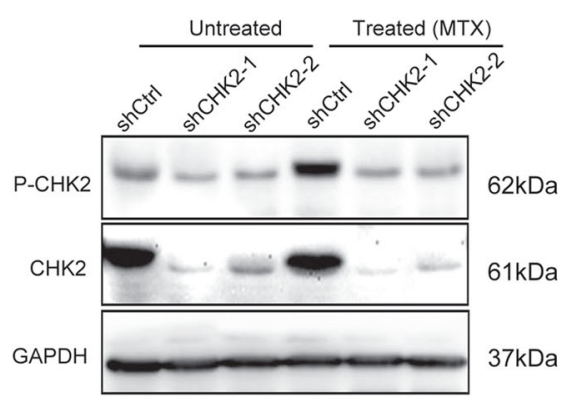

C

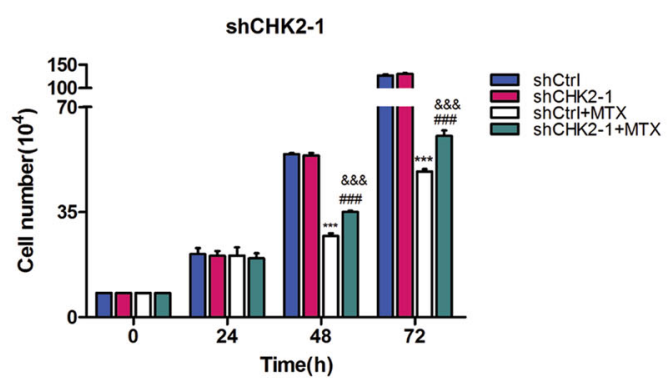

d

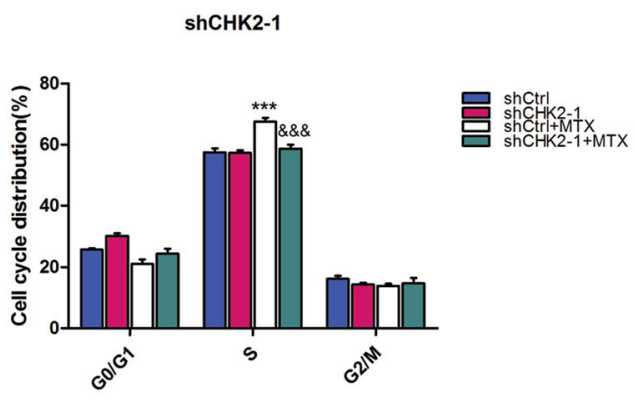

e

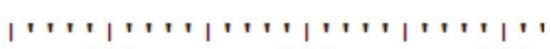

190

200

210

CHK2(WT) CCTTAGAGACAGTGTCC CTCAGGAACT CHK2(T68A) CCTTAGAGACAGTGTCCGCT)CAGGAACT

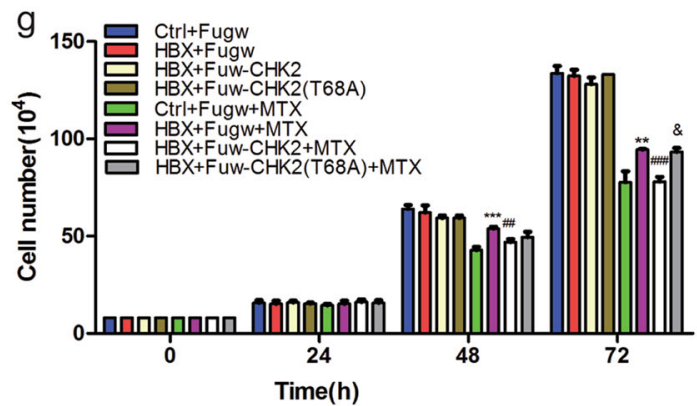

b

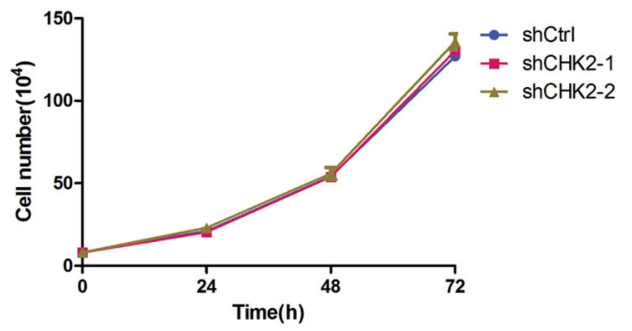

shCHK2-2

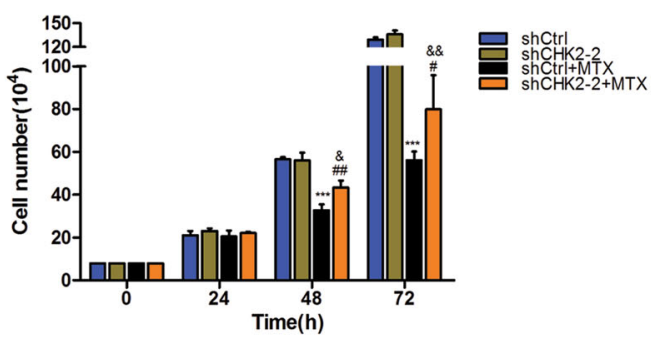

shCHK2-2
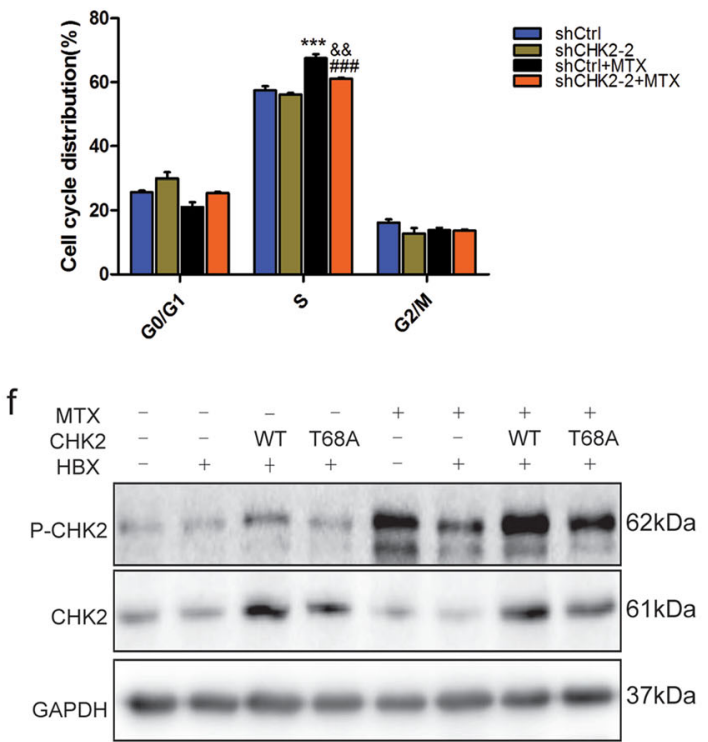

h

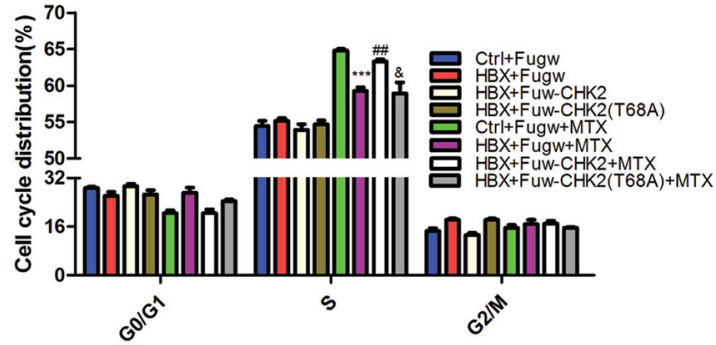

Fig. 4 (See legend on next page.) 


\begin{abstract}
(see figure on previous page)
Fig. 4 CHK2 phosphorylation rescues chemosensitivity in HBX-expressing cells. a The expression of CHK2 and P-CHK2 was measured by western blotting upon CHK2 depletion by two independent shRNAs with or without MTX treatment. b Depletion of CHK2 showed no significant effect on the proliferation of DLBCL cells. c Cell-counting assays for control, shCHK2-1 and shCHK2-2 SUDHL-4 cells with or without MTX (4 ng/ml) treatment at 24,48 , and $72 \mathrm{~h}$. d Quantification of the cell cycle distributions of control and HBX-expressing cells with or without MTX treatment for $48 \mathrm{~h}$. The results are shown as the mean \pm SEM from triplicate experiments. " $*{ }^{* \prime}$ Represents shCtrl + MTX versus shCtrl: ${ }^{* *} P<0.01,{ }^{* * *} P<0.001$. "\#" Represents shCHK2 + MTX versus shCHK2: ${ }^{\# \#} P<0.01,{ }^{\# \#} P<0.001$. " $\&$ " Represents shCHK2 + MTX versus shCtrl + MTX: ${ }^{{ }^{2}} p<0.05$, ${ }^{\text {\& }} P<0.01,{ }^{\& \& \&} p<0.001$. e The schematic diagram of wild-type and mutant (T68A) CHK2. f Western blotting analysis for CHK2 and P-CHK2 in control and HBX-expressing cells transfected with Fuw (empty vector), Fuw-CHK2 (WT), or Fuw-CHK2 (T68A) with or without MTX treatment. GAPDH was used as a loading control. g, $\mathbf{h}$ Cell counting $\mathbf{g}$, and cell cycle distribution analysis $\mathbf{h}$, of the indicated groups with or without MTX treatment. The results are shown as the mean \pm SEM from triplicate experiments. " "*" Represents HBX + MTX versus Ctrl + MTX: ** $P<0.01$, *** $P<0.001$. "\#" Represents HBX + CHK2 (WT) + MTX versus HBX + MTX: ${ }^{\# \#} P<0.01,{ }^{\# \# \#} P<0.001$. " $\&$ " Represents HBX + CHK2(T68A) + MTX versus HBX + CHK2(WT) + MTX: ${ }^{\&} P<0.05$
\end{abstract}

pancreatic cancer ${ }^{44,45}$ and to activate CHK2 phosphorylation to delay the cell cycle in hepatocarcinogenesis ${ }^{46}$, suggesting that the direct regulation of protein phosphorylation is an important mechanism for HBX in carcinogenesis. Our findings first indicated that $\mathrm{HBX}$ specifically inhibited CHK2 phosphorylation, which conferred resistance to chemotherapeutics that induced Sphase arrest in DLBCL.

Evidence has shown that combining cytotoxic chemotherapeutics with pharmacological CHK2 inhibitors can prevent damaged cancer cells from arresting and improve therapeutic efficacy ${ }^{25,47,48}$. CHK2 inhibitors have already entered phase I \& II clinical trials ${ }^{48,49}$. However, it has also been reported that CHK2 depletion reduces the chemosensitivity of various DNA-damaging agents including MTX in the treatment of Burkitt lymphoma ${ }^{24}$. High expression of CHK2 also results in a better response to platinum-based chemotherapy in ovarian cancer ${ }^{25}$. Our results showed that HBV infection caused resistance to Sphase arrest-inducing drugs in DLBCL by suppressing CHK2 phosphorylation, suggesting that HBV-infected DLBCL patients might not benefit from the use of CHK2 inhibitors.

In summary, our study uncovered the association between HBV infection and resistance to MTX- or Ara-Cbased chemotherapy mediated by the inhibition of CHK2 response signaling, which provides new insight into the role of HBV in the chemoresistance of DLBCL patients. Therefore, HBV-infected DLBCL patients may benefit less from the use of S-phase arrest-inducing chemotherapeutics or CHK2 inhibitors. Further prospective investigations utilizing HBX-directed targeted therapy may help manage this challenge.

\section{Materials and methods \\ Patients}

We collected clinical data (name, gender, stages, international prognostic index (IPI) score, cell of origin, Ki67 expression, LDH, chemotherapy regiments, therapeutic evaluation, HBsAg status, liver indicator, OS, and PFS) of 428 cases DLBCL patients with or without HBV infection from January 2004 to July 2014 in Changhai Hospital, Shanghai, China. This study was approved by the institutional review board of the Changhai Hospital. The diagnosis of B-cell lymphoma used current World Health Organization (WHO) classification criteria. HBV infection status was determined by HBsAg. Stage criteria were based on the Ann Arbor staging criteria. The treatment efficacy was evaluated according to WHO curative standard.

\section{Cell culture and chemotherapeutics}

Human 293T cells and DLBCL cell lines (SUDHL-4 and DB) were purchased from the American Type Culture Collection (ATCC) and cultured in Dulbecco's modified Eagle's medium containing $10 \%$ fetal bovine serum (Gibco) at $37^{\circ} \mathrm{C}$ with $5 \% \mathrm{CO}_{2}$ in a humidified incubator. The following chemotherapeutics were used: MTX (Pfizer, Shanghai, China), Ara-C (Pharmacia, Shanghai, China), EPI (Hisun, Hangzhou, China), and VDS (Minsheng, Hangzhou, China).

\section{Plasmid construction and stable transfection}

The DNA fragments of HBX gene cloned from HepG2.2.15 cells were inserted into the pLVX-3 $\times$ flagIRES-ZsGreen1 vector, and the CHK2 DNA fragments cloned from SUDHL-4 cells were inserted into the Fuw vector. The CHK2 shRNAs were inserted into the pLKO.1 vector. Plasmid transfection was performed according to protocols supplied with the X-tremeGENE (Roche, Indianapolis, USA). Viral supernatant harvested $48 \mathrm{~h}$ after transfection was used to infect DLBCL cells in the presence of polybrene $(8 \mu \mathrm{g} / \mathrm{ml})$. Infected cells were selected with puromycin (Invitrogen, Carlsbad, CA, USA) at a final concentration of $2 \mu \mathrm{g} / \mathrm{ml}$. The primers are shown in Supplementary Table S1.

\section{Cell growth assays and cell cycle analysis}

Cells $\left(8 \times 10^{4}\right)$ were seeded in a 12 -well plate, and the indicated amounts of chemotherapeutics were added. Cell numbers were measured by Cell Counter Star after 24, 48, and $72 \mathrm{~h}$. The cells were harvested at $48 \mathrm{~h}$ and fixed in 1 


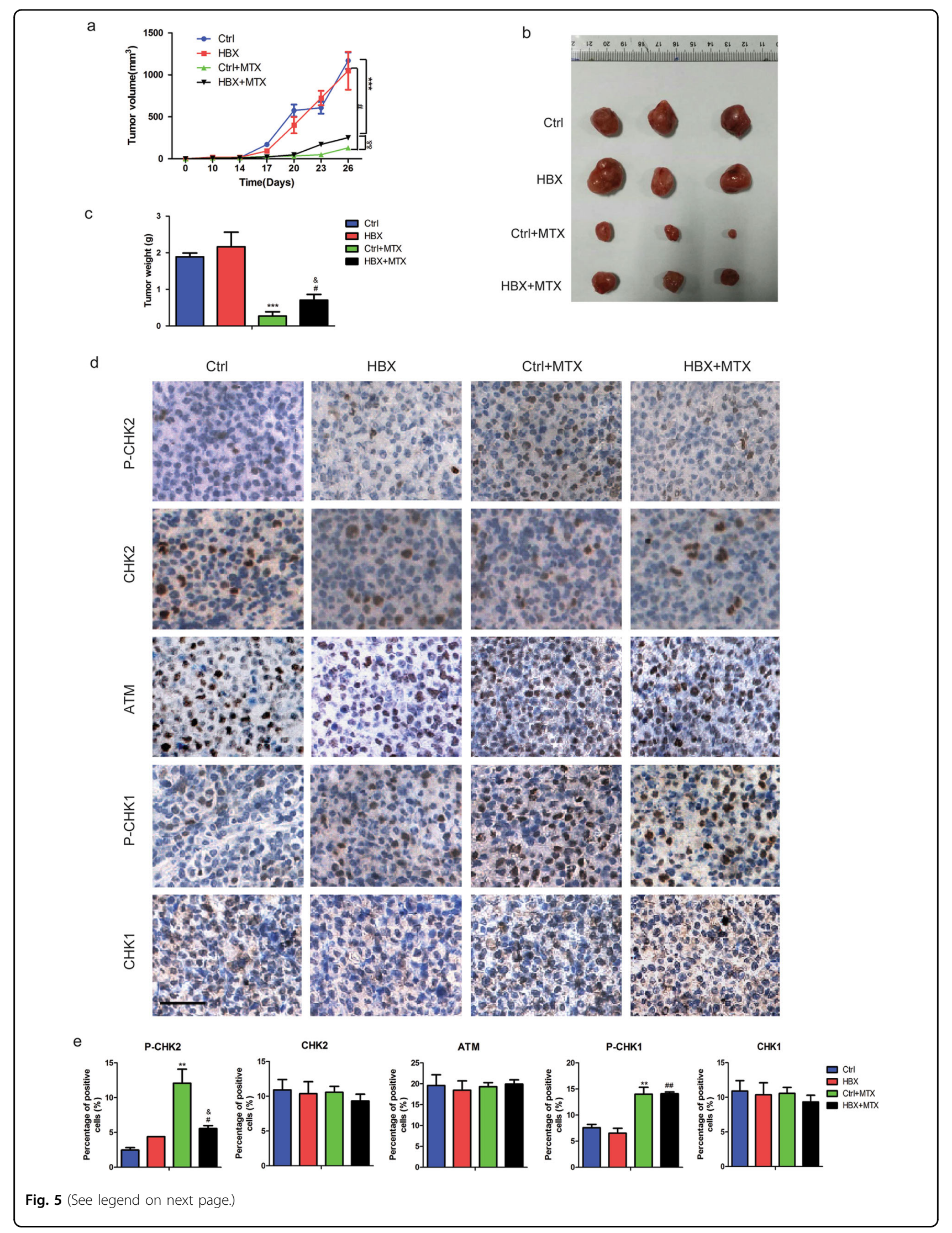


(see figure on previous page)

Fig. 5 HBX confers resistance to chemotherapy in vivo. a Tumor volumes were measured every 3 days after day 14 post-injection in the indicated groups ( $n=3$ per group). b, $\mathbf{c}$ Representative images $\mathbf{b}$, and weight statistics $\mathbf{c}$, of tumors isolated from the mice for one of the batches at day 26 post-injection. $\mathbf{d}$ Immunohistochemical analysis of tumor sections from four groups using the indicated antibodies (P-CHK2, CHK2, ATM, P-CHK1, and CHK1). Scale bar, $25 \mu \mathrm{m}$. e Histogram statistics for the cells positive for the indicated proteins are shown. "** Represents Ctrl + MTX versus Ctrl: **P< 0.01 , ${ }^{* * *} P<0.001$. "\#" Represents HBX + MTX versus HBX: ${ }^{*} P<0.05$, ${ }^{\# \# ~} P<0.01$. " $\&$ " Represents HBX + MTX versus Ctrl + MTX: ${ }^{\&} P<0.05$, \& $P<0.01$

$\mathrm{ml}$ of pre-cooled $70 \%$ ethanol for at least $8 \mathrm{~h}$. The cells were stained with propidium iodide/RNase (Beyotime, Shanghai, China) at $37^{\circ} \mathrm{C}$ for $30 \mathrm{~min}$ in the dark. Cell cycle distributions were evaluated by flow cytometry using a FACS Calibur (Becton Dickinson Biosciences, Franklin Lakes, NJ, USA) and analyzed by FlowJo software.

\section{Western blotting and immunohistochemistry}

Western blotting and immunohistochemistry were performed according to standard procedures ${ }^{50}$. The following antibodies were used: anti-HBX (ab39716, Abcam, Cambridge, MA, USA), anti-P-CHK2 (2197S, Cell Signaling Technology, Danvers, MA, USA), anti-CHK2 (ab47433, Abcam), anti-ATM (ab199726, Abcam), anti-P21 (ab7960, Abcam), anti-P53 (BS1913, Bioworld, Minnesota, USA), anti- $\gamma$ H2AX (ab26350, Abcam), anti-P-CHK1 (2348S, Cell Signaling Technology), anti-CHK1 (10362-1-AP, Proteintech), anti-CDK2 (SC-6248, Santa Cruz Biotechnology, CA, USA), anti-Cyclin D1 (SC-718, Santa Cruz Biotechnology), anti-GAPDH (SC-47724, Santa Cruz Biotechnology), HRP-Ms (\#7074, Cell Signaling Technology), and HRP-Rb (\#7076, Cell Signaling Technology).

\section{Quantitative RT-PCR (qPCR)}

Total RNA was extracted from the cells using RNAiso (9109, Takara, Japan). First-strand complementary DNA synthesis was performed using the PrimeScript ${ }^{\text {TM }}$ RT reagent kit (RR037A, Takara). The qPCR analysis was performed using SYBR Premix Ex Taq TM (RR420A, Takara) with an Agilent Stratagene Mx3000P instrument. The GAPDH gene was employed as an internal control. The primer sequences used in this study are shown in Supplementary Table S2.

\section{Tumor xenografts}

Five-week-old NOD-SCID mice were purchased from the National Resource Center for Rodent Laboratory Animals of China. Control and HBX-expressing DB cells $\left(1 \times 10^{7}\right.$ each), suspended in $100 \mu \mathrm{l}$ of one part Matrigel and two parts DMEM, were subcutaneously injected into the left and right thigh of mice, respectively. On day 14 after tumor cells injection, the mice were monitored to assess the tumor volume using the formula $1 / 2$ (length $\times$ width $^{2}$ ) and injected intraperitoneally with MTX (10 mg/ $\mathrm{kg}$ ) every 3 days until day 26 . The mice were sacrificed on day 26 post-injection. All experiments were carried out as approved by the Institutional Animal Care and Use Committee of Tongji University.

\section{Statistical analysis}

The Kaplan-Meier method and log-rank test were used to generate and compare the OS and PFS between different groups. We used the chi-square test and nonparametric test to compare categorical variables. The statistics were prepared using Excel and GraphPad Prism 5 software. The experiments were performed in triplicate and all data were presented as the mean \pm standard error of mean (SEM). Normally distributed groups were compared by two-tailed Student's $t$-test. $P<0.05$ was considered statistically significant.

\section{Acknowledgements}

This work was supported by projects of the National Natural Science Foundation of China (81570179 and 81170499 to J. C).

\section{Author details}

${ }^{1}$ Department of Hematology, Changhai Hospital, Second Military Medical University, Shanghai, China. ${ }^{2}$ Clinical and Translational Research Center of Shanghai First Maternity and Infant Health Hospital, Shanghai Key Laboratory of Signaling and Disease Research, Collaborative Innovation Center for Brain Science, School of Life Science and Technology, Tongji University, Shanghai, China. ${ }^{3}$ Institute of Regenerative Medicine, East Hospital, Tongji University School of Medicine, Shanghai, China. ${ }^{4}$ Department of Hematology, Jingzhou Central Hospital, Jingzhou Clinical Medical College, Yangtze University, Jingzhou, China. ${ }^{5}$ Department of Pathology, Changhai Hospital, Second Military Medical University, Shanghai, China

\section{Conflict of interest}

The authors declare that they have no conflict of interest.

\section{Supplementary Information}

The online version of this article (https://doi.org/10.1038/s41419-017-0097-1) contains supplementary material.

Received: 13 September 2017 Revised: 23 October 2017 Accepted: 23 October 2017

Published online: 19 January 2018

\section{References}

1. Sehn, L. H. \& Gascoyne, R. D. Diffuse large B-cell lymphoma: optimizing outcome in the context of clinical and biologic heterogeneity. Blood 125, 22-32 (2015).

2. Pfreundschuh, M. et al. CHOP-like chemotherapy plus rituximab versus CHOPlike chemotherapy alone in young patients with good-prognosis diffuse largeB-cell lymphoma: a randomised controlled trial by the MabThera International Trial (MInT) Group. Lancet Oncol. 7, 379-391 (2006). 
3. Coiffier, B. et al. Long-term outcome of patients in the LNH-98.5 trial, the first randomized study comparing rituximab-CHOP to standard $\mathrm{CHOP}$ chemotherapy in DLBCL patients: a study by the Groupe d'Etudes des Lymphomes de l'Adulte. Blood 116, 2040-2045 (2010).

4. zur Hausen, $H$. et al. EBV DNA in biopsies of Burkitt tumours and anaplastic carcinomas of the nasopharynx. Nature 228, 1056-1058 (1970).

5. Marcucci, F. \& Mele, A. Hepatitis viruses and non-Hodgkin lymphoma: epidemiology, mechanisms of tumorigenesis, and therapeutic opportunities. Blood 117, 1792-1798 (2011).

6. Chen, J. et al. Concurrent infection of hepatitis B virus negatively affects the clinical outcome and prognosis of patients with non-Hodgkin's lymphoma after chemotherapy. PLOS ONE 8, e69400 (2013).

7. Kim, J. H. et al. Hepatitis B virus infection and B-cell non-Hodgkin's lymphoma in a hepatitis B endemic area: a case-control study. Japanese journal of cancer research. Jpn. J. Cancer Res. 93, 471-477 (2002).

8. Takai, S. et al. Prevalence of hepatitis B and C virus infection in haematological malignancies and liver injury following chemotherapy. Eur. J. Haematol. 74, 158-165 (2005)

9. Deng, L. et al. Hepatitis B virus-associated diffuse large B-cell lymphoma: unique clinical features, poor outcome, and hepatitis B surface antigen-driven origin. Oncotarget 6, 25061-25073 (2015).

10. Srisuttee, R. et al. SIRT1 sensitizes hepatocellular carcinoma cells expressing hepatitis $B$ virus $X$ protein to oxidative stress-induced apoptosis. Biochem. Biophys. Res. Commun. 429, 45-50 (2012).

11. Chen, $Z$ et al. HBx mutations promote hepatoma cell migration through the Wnt/beta-catenin signaling pathway. Cancer. Sci. 107 1380-1389 (2016)

12. Gao, B., Wang, H., Lafdil, F. \& Feng, D. STAT proteins - key regulators of anti-viral responses, inflammation, and tumorigenesis in the liver. J. Hepatol. $\mathbf{5 7}$ 430-441 (2012).

13. Kong, F. et al. The enhanced expression of death receptor 5 (DR5) mediated by HBV X protein through NF-kappaB pathway is associated with cell apoptosis induced by (TNF-alpha related apoptosis inducing ligand) TRAIL in hepatoma cells. Virol. J. 12, 192 (2015).

14. Pontisso, P., Vidalino, L., Quarta, S. \& Gatta, A. Biological and clinical implications of HBV infection in peripheral blood mononuclear cells. Autoimmun. Rev. 8 13-17 (2008).

15. Wang, F. et al. High hepatitis B virus infection in B-cell lymphoma tissue and its potential clinical relevance. Eur. J. Cancer Prev. 21, 261-267 (2012).

16. El-Sayed, G. M., Mohamed, W. S., Nouh, M. A., Moneer, M. M. \& El-Mahallawy, H. A. Viral genomes and antigen detection of hepatitis $B$ and $C$ viruses in involved lymph nodes of Egyptian non-Hodgkin's lymphoma patients. Egyptian J. Immunol. 13, 105-114 (2006).

17. Umeda, M. et al. Hepatitis B virus infection in lymphatic tissues in inactive hepatitis B carriers. J. Hepatol. 42, 806-812 (2005).

18. Kwok, M. et al. Synthetic lethality in chronic lymphocytic leukaemia with DNA damage response defects by targeting the ATR pathway. Lancet (London, England) 385(Suppl 1), S58 (2015)

19. Bartek, J. \& Lukas, J. Chk1 and Chk2 kinases in checkpoint control and cancer. Cancer Cell. 3, 421-429 (2003).

20. Chehab, N. H., Malikzay, A., Stavridi, E. S. \& Halazonetis, T. D. Phosphorylation of Ser-20 mediates stabilization of human p53 in response to DNA damage. Proc. Natl. Acad. Sci. USA 96, 13777-13782 (1999).

21. Chehab, N. H., Malikzay, A., Appel, M. \& Halazonetis, T. D. Chk2/hCds1 functions as a DNA damage checkpoint in $\mathrm{G}(1)$ by stabilizing p53. Genes Dev. 14, 278-288 (2000)

22. Lukas, C., Falck, J., Bartkova, J., Bartek, J. \& Lukas, J. Distinct spatiotempora dynamics of mammalian checkpoint regulators induced by DNA damage. Nat. Cell. Biol. 5, 255-260 (2003).

23. Kastan, M. B. \& Bartek, J. Cell-cycle checkpoints and cancer. Nature 432 316-323 (2004).

24. Jiang, H., Pritchard, J. R., Williams, R. T., Lauffenburger, D. A. \& Hemann, M. T. A mammalian functional-genetic approach to characterizing cancer therapeutics. Nat. Chem. Biol. 7, 92-100 (2011).

25. Martin, L. P., Hamilton, T. C. \& Schilder, R. J. Platinum resistance: the role of DNA repair pathways. Clin. Cancer Res. 14, 1291-1295 (2008).
26. Squatrito, M. et al. Loss of ATM/Chk2/p53 pathway components accelerates tumor development and contributes to radiation resistance in gliomas. Cancer Cell. 18, 619-629 (2010).

27. Cha, $\mathrm{H}$. et al. Wip1 directly dephosphorylates gamma-H2AX and attenuates the DNA damage response. Cancer Res. 70, 4112-4122 (2010).

28. Bonner, W. M. et al. GammaH2AX and cancer. Nat. Rev. Cancer 8, 957-967 (2008).

29. Cannell, I. G. et al. A pleiotropic RNA-binding protein controls distinct cell cycle checkpoints to drive resistance of p53-defective tumors to chemotherapy. Cancer Cell. 28, 623-637 (2015)

30. Alkema, N. G. et al. Checkpoint kinase 2 (Chk2) supports sensitivity to platinum-based treatment in high grade serous ovarian cancer. Gynecol. Oncol. 133, 591-598 (2014).

31. Engels, E. A., Cho, E. R. \& Jee, S. H. Hepatitis B virus infection and risk of nonHodgkin lymphoma in South Korea: a cohort study. Lancet Oncol. 11, 827-834 (2010).

32. Mathur, R. et al. Inhibition of demethylase KDM6B sensitizes diffuse large B-cell Iymphoma to chemotherapeutic drugs. Haematologica 102, 373-380 (2016).

33. Kim, D. H. et al. FCGR3A gene polymorphisms may correlate with response to frontline R-CHOP therapy for diffuse large B-cell lymphoma. Blood $\mathbf{1 0 8}$ 2720-2725 (2006)

34. Chen, L. et al. SYK-dependent tonic B-cell receptor signaling is a rational treatment target in diffuse large B-cell lymphoma. Blood 111, 2230-2237 (2008).

35. Petrich, A. M. et al. Impact of induction regimen and stem cell transplantation on outcomes in double-hit lymphoma: a multicenter retrospective analysis. Blood 124, 2354-2361 (2014).

36. Chihara, D. et al. Dose-adjusted EPOCH-R and mid-cycle high dose methotrexate for patients with systemic lymphoma and secondary CNS involvement. Br. J. Haematol 179, 851-854. (2016).

37. Purroy, $\mathrm{N}$. et al. Long-term follow-up of dose-adjusted EPOCH plus rituximab (DA-EPOCH-R) in untreated patients with poor prognosis large B-cell lymphoma. A phase II study conducted by the Spanish PETHEMA Group. Br. J. Haematol. 169, 188-198 (2015).

38. Rizzetto, M. \& Ciancio, A. Chronic HBV-related liver disease. Mol. Aspects. Med. 29, 72-84 (2008).

39. Shi, Y. H. \& Shi, C. H. Molecular characteristics and stages of chronic hepatitis B virus infection. World J. Gastroenterol. 15, 3099-3105 (2009).

40. Benn, J. \& Schneider, R. J. Hepatitis B virus HBx protein activates Ras-GTP complex formation and establishes a Ras, Raf, MAP kinase signaling cascade. Proc. Natl. Acad. Sci. USA 91, 10350-10354 (1994).

41. Benn, J. \& Schneider, R. J. Hepatitis B virus HBx protein deregulates cell cycle checkpoint controls. Proc. Natl. Acad. Sci. USA 92, 11215-11219 (1995).

42. Carroll, B. L., Pulkoski-Gross, M. J., Hannun, Y. A. \& Obeid, L. M. CHK1 regulates NF-kappaB signaling upon DNA damage in p53- deficient cells and associated tumor-derived microvesicles. Oncotarget 7, 18159-18170 (2016).

43. Wang, L., Mosel, A. J., Oakley, G. G. \& Peng, A. Deficient DNA damage signaling leads to chemoresistance to cisplatin in oral cancer. Mol. Cancer Ther. 11, 2401-2409 (2012).

44. Chen, $Y$. et al. The hepatitis B virus $X$ protein promotes pancreatic cancer through modulation of the PI3K/AKT signaling pathway. Cancer Lett. $\mathbf{3 8 0}$, 98-105 (2016).

45. Han, M. et al. Hepatitis B virus-induced hFGL2 transcription is dependent on CEts-2 and MAPK signal pathway. J. Biol. Chem. 283, 32715-32729 (2008).

46. Kim, S. et al. Hepatitis B virus $X$ protein activates the ATM-Chk2 pathway and delays cell cycle progression. J. Gen. Virol. 96, 2242-2251 (2015).

47. Carlessi, L. et al. Biochemical and cellular characterization of VRX0466617, a novel and selective inhibitor for the checkpoint kinase Chk2. Mol. Cancer Ther. 6, 935-944 (2007)

48. Garrett, M. D. \& Collins, I. Anticancer therapy with checkpoint inhibitors: what, where and when? Trends Pharmacol. Sci. 32, 308-316 (2011).

49. Lord, C. J., Garrett, M. D. \& Ashworth, A. Targeting the double-strand DNA break repair pathway as a therapeutic strategy. Clin. Cancer Res. 12, 4463-4468 (2006).

50. Rijal, S. et al. Inositol polyphosphate 4-phosphatase ॥ (INPP4B) is associated with chemoresistance and poor outcome in AML. Blood 125, 2815-2824 (2015). 